reated in our clinic for the 6-years (1960-1965).

Of all the cases 104 were death ones. According to the classification of lead injury (by Araki, 1954), simple type were 1888 cases, commotion type 1782 zases, contusion type 588 cases, intracranial haematoma type 85 cases and 24 zases were unknown. The interval from injury to medical examination was classihied as follows: within 3 days after trauma were 2188 cases, 4 to 7 days after 306 cases (subacute) and over 8 days after (chronic) was 187 cases. In the clinicostatistical studies on our cases, the death cases tended to concentrate in the following three critical periods: the first was immediately after trauma (initial shock), the second about 3 days after (increased intracranial pressure), the third one week after (secondary complications, chronic hypoxia and metabolic unbalance). Except for the critical periods, the symptoms and the signs of patients were comparatively stable.

Moreover, the detailed analysis was performed of the clinical informations on 653 admitted patients. Being compared with the admitted patients of the same type (by Araki) before 1962, more severe ones increased after 1963. Follow-up study of the prognosis of the 43 patients with triad (absence of lucid intervals, coma and anisocoria) showed the following interesting results. The 7 patients of the 13 patients surgically treated died (mortality rate was $53.9 \%$ ), the 9 patients of the 34 cases conservatively managed died (mortality rate $26.5 \%$ ).

In short, it is suggested through our clinico-statistical observations that conservative management should be done for the patients with triad, unless large intracranial haematoma is evidently demonstrated by angiography.

Table 1 show higher mortality, Table 2 lower mortality.

\title{
134. Experiences of Emergent Cases of Head Injuries
}

\author{
Hideyuki HiraI \\ Dept. of Neurosurgery, Kanagawa Ambulance Center \\ Yoshiyuki Shibata, Masaki Moriyama and Ryozo Fujinaga \\ Dept. of Neurosurgery, Keio University
}

\section{Statistic Observation of Head Injuries (866 Cases seen Between 1965 and 1967)}

\author{
Yoshiro TaKeUCHI \\ Neurosurgical Service, Handa City Hospital, Handa City, Aichi
}

